\title{
El marketing holístico en la oferta de prestación de servicios de salud en Colombia
}

\author{
JUAN MANUEL CÁRDENAS MUNÉVAR
}

\section{RESUMEN}

Este texto insinúa una relación de causalidad entre el marketing holístico, el mejoramiento de los niveles de calidad y el aumento en la eficiencia de los procesos de prestación de servicios de salud en Colombia.

El texto presenta inicialmente, la conceptualización de "mercadotecnia sanitaria", luego la situación de la salud en Colombia anterior a la expedición y la puesta en marcha de la Ley 100 de 1993 y del Sistema de Salud derivado de la misma. Posteriormente, conceptualiza sobre holismo y Marketing Holístico, citando a Jan Smuts, y fragmentos de una entrevista a Philip Kotler. Finalmente deja, de alguna forma, la inquietud en el lector sobre qué tanto ha incidido el marketing holístico en la ampliación de la cobertura en la prestación de servicios de salud, mostrando en cifras la situación de los últimos seite años, de 2000 a 2006.

\section{PALABRAS CLAVE}

Mercadotecnia sanitaria, holismo, holístico, marketing holístico, Instituto de Seguros Sociales ISS, Sistema Nacional de Salud SNS, Planeación de los Recursos de una Empresa ERP, Gestión de Cadena de Abastecimiento SCM , Gestión de Relaciones con el cliente CRM.

\section{La mercadotecnia sanitaria}

El marketing, a través del tiempo, ha sido adoptado y aplicado con especificidad, ya sea en el sector industrial, en el sector de consumo o en el de servicios. En este último, cada vez hay más conciencia de la importancia del marketing en el desarrollo de la actividad.

Hoy en día se habla de un concepto nuevo: la "mercadotecnia sanitaria" 1 .

El doctor Heberto Priego Álvarez ${ }^{2}$ en su ensayo "Mitos y realidades en la mercadotecnia de servicios de salud", nos explica cómo los usos de la mercadotecnia sanitaria pueden ser externos o internos a la empresa de salud. En el ámbito externo permite mejorar la imagen del producto o del servicio de salud, atrae recursos y personas hacia la institución, y regula el nivel de la demanda, estimulándola o revitalizándola; o por el contrario, desincentivando un uso o un consumo excesivo haciendo lo que se conoce como desmercadotecnia o contramercadotecnia (demarketing). Internamente, se aplica para hacer un mejor uso de los recursos existentes, en el desarrollo de nuevos servicios y programas de salud y, para motivar y comprometer a los empleados con la misión y el objetivo de la organización, procurando la mejoría en los aspectos humanos o interpersonales de la calidad. La mercadotecnia interna debe entenderse como un "atrayente o incentivador que retiene a unos clientes internos por medio de unos productos-trabajos destinados a satisfacer sus necesidades y apetencias" (Lamata et al., 1994:195). Al respecto es conveniente considerar que una empresa de salud aumenta su capacidad para satisfacer las necesidades y deseos de sus clientes externos (consumidores, usuarios, aseguradoras), si satisface previamente la de sus clientes internos (trabajadores, profesionales).

1 William L. Pierpoint, presidente y director ejecutivo de Summit Health Limited, organización sanitaria lucrativa con más de 5,500 camas en cinco estados del suroeste de los Estados Unidos y en el extranjero.

2 Priego Álvares, Heberto. Mitos y realidades en la mercadotecnia de servicios de salud. Salud Tab 2001: 7(2): 408-413. Marketing en salud. En: http://www.gerenciasalud.com. 
La mercadotecnia en salud es un ejercicio profesional y una disciplina administrativa cada vez más tecnificada, cuya esencia radica en que esta se vincula directamente a la gestión de cualquier institución de salud (clínica, hospital, etcétera) y la orienta (March-Cerda et al., 1996). Esta orientación tiene como clave la determinación de las necesidades y valores de la población objetivo (mercado -meta) con el fin de conseguir el deseado nivel de satisfacción. Por eso, las actuales empresas de salud son más sensibles a las necesidades, preferencias y expectativas de los usuarios para satisfacerlas (investigación de mercado), estableciendo estrategias diversas en función de públicos distintos (segmentación de mercado); ofertando un conjunto de actividades que tengan como base la calidad (marketing mix) y desarrollando una ventaja competitiva (diferenciación, posicionamiento, imagen institucional y merchandising).

La calidad de un servicio, tal y como es percibido por los clientes de la salud tiene dos dimensiones, una técnica o de resultado y una funcional o relacionada con el proceso (Grönroos, 1983; Parasuraman et al., 1985; Lethninen, 1986). La calidad técnica del resultado del proceso productivo del servicio no es medible con suficiente objetividad por los usuarios debido a su carácter de solución técnica con respecto a un problema de salud y por la asimetría en la información existente entre prestador y prestatario.

La mejora en las interacciones proveedor-usuario se convierte en la base de los programas de calidad.

La principal ventaja competitiva de los servicios de salud radica en su calidad funcional o relacionada con

\section{Juan Manuel Cárdenas Munévar}

Especialista en Gerencia de Mercadeo, Administrador Financiero, Administrador de Empresas, miembro del grupo de investigación del Programa de Mercadeo y Publicidad del Politécnico Grancolombiano Institución Universitaria . Consultor empresarial. Para contactar al autor: jcardena@poligran.edu.co

|

3 Real Academia Española. Diccionario de la Lengua Española. $22^{\circ}$ edición. el proceso, afirmación asociada a la prestación de los servicios médicos:

La calidad sanitaria significa una distribución experta y eficaz del servicio de salud no sólo en el sentido técnico sino en el personal. Por tanto, Summit se ha centrado en programas de formación y educación de sus empleados, en los que se resalta la importancia de las relaciones con los pacientes. ( $\cdots)$ esta inversión en calidad funcional no producirá beneficios de inmediato, pero actuará a largo plazo, como un apoyo a la consolidación de la ventaja competitiva. (Grönroos, 1994:40-41).

Se ve claramente la relación que se establece entre el entorno externo y el interno, buscando en el primero mejorar la percepción del producto o servicio de salud, optimizando los procesos, buscando mejor personal en el mercado laboral y buscando, de otra parte, la adquisición de nuevos clientes y su fidelización.

En la empresa se busca la alineación de la cultura organizacional, la tecnología de información y comunicaciones y el capital intelectual (el recurso humano), con un objetivo corporativo único de mejorar los aspectos humanos e interpersonales de la calidad, apoyados en procesos tecnológicos internos no perceptibles o medibles fácilmente por los usuarios.

Los planteamientos de Summit cuando conceptualiza sobre mercadotecnia sanitaria, nos acercan al tema de trabajo en equipo y a los procesos sinérgicos que permiten la agregación de valor tanto para la entidad como para los usuarios, y además nos acercan al concepto de marketing holístico.

Holismo: (De holo- e -ismo). Es la doctrina que propugna por la concepción de cada realidad como un todo distinto de la suma de las partes que lo componen ${ }^{3}$.

Es la idea de que todas las propiedades de un sistema (biológico, químico, social, económico, mental, lingüístico, etc.) no pueden ser determinadas o explicadas como la suma de sus componentes. El sistema completo se comporta de un modo distinto a la suma de sus partes. 
Se puede definir como el tratamiento de un tema que implica a todos sus componentes, con sus relaciones obvias e invisibles. Normalmente se usa como una tercera vía o nueva solución a un problema. El holismo hace énfasis en la importancia del todo, pues es más grande que la suma de las partes, y a la interdependencia de las partes.

\section{Holístico}

Los conceptos científicos, han cambiado a la par con la evolución del hombre; en la ciencia lo definitivo no existe; para conocer lo que existe es necesario saber cuál es su origen, cuál su significado y cómo funciona; un conglomerado de materia no conforma nada por sí solo; las interrelaciones de los elementos son los que dan sentido a lo que tenemos.

Ernesto Iturralde en el artículo "El Pensamiento Lateral, Vertical y Holístico" 4 nos introduce en el concepto de "holismo" como palabra que describe la tendencia de la naturaleza a crear conjuntos mediante el ordenamiento o agrupación de muchas unidades, y data la aparición de la palabra en 1927 citando a Jan Smuts ${ }^{5}$, experto agrónomo y militar quien acuñó el término "holismo" en su libro Holism and Evolution.

El pensamiento holístico percibe las cosas en su conjunto y no analiza sus partes. Según Smuts, las realidades básicas naturales son conductos irreductibles inseparables para analizarlos según sus componentes sin perder su cualidad "holística".

Por ejemplo, el trabajo en equipo es un concepto holístico con resultados sinérgicos.

Adentrándonos aún más en el concepto, es importante ver cómo la internet, la globalización y la hipercompetencia están redefiniendo profundamente los mercados y cambiando la manera de actuar en los negocios. Sin embargo, opinan algunos autores, que el marketing no ha avanzado al mismo ritmo de los mercados. Ante esto es necesario, dice Kotler ${ }^{6}$, redefinir y ampliar el marketing clásico para reflejar esa nueva realidad. Él señala que todo esto exige un replanteamiento fundamental de la estrategia empresarial para permitir la creación de valores añadidos para los clientes, tanto en el mercado como en el espacio mercantil. Y es el marketing el que se convierte en principal motor del desarrollo y de la aplicación de esta nueva estrategia. ¿Cómo lograrlo? Utilizar un paradigma del marketing radicalmente nuevo llamado marketing holístico, un concepto dinámico derivado del entorno electrónico y de la interactividad entre compañías, clientes y colaboradores. Este nuevo paradigma combina lo mejor del marketing tradicional con las nuevas capacidades digitales para desarrollar relaciones a largo plazo mutuamente satisfactorias. Perfilar un esquema para llevar a la práctica el marketing holístico requiere integrar la gestión de la demanda del cliente, la asignación de recursos internos y externos, y la colaboración sistemática entre unidades.

El marketing holístico puede ayudar a las compañías a: identificar las oportunidades para renovar sus mercados. Crear eficientemente las mejores ofertas. Ofrecer productos y servicios que respondan a las necesidades del cliente. Operar en el más alto nivel de calidad del producto en cuanto a servicio y velocidad. Desarrollar una plataforma de marketing completa, lista para los desafíos y oportunidades que surgen de un mundo empresarial centrado en el cliente.

\section{Pero ¿qué es un enfoque holístico del marketing?}

El marketing ha sido tratado en las empresas a menudo como un departamento, uno que esencialmente se ocupa de las comunicaciones y promociones. En el libro Marketing Moves ${ }^{7}$ los autores argumentan que el marketing, adecuadamente concebido, es una función estratégica y debiera ser el conductor de la estrategia de la empresa. El enfoque holístico, entonces, demanda al marketing convertirse en el arquitecto de las cadenas de demanda y suministros de la empresa y de su red de colaboradores.

En una entrevista para Clarín.com periodismo en internet, en el suplemento económico de junio de 2001 ("Cómo crear valor para el cliente"), se le preguntó a Kotler:"-Usted anuncia la llegada de un cambio, un nuevo paradigma, ¿cuál había sido el anterior y cómo se hace para pasar del antiguo al nuevo esquema?"

La respuesta de Kotler nos ubica de forma mas concreta en el concepto:

4 Iturralde, Ernesto. "El pensamiento lateral, vertical y holístico". En: http://www.yturralde.com/plateral.htm. 2007.

5 El Mariscal Jan Christiaan Smuts, (24 de mayo de 1870 - 11 de septiembre de 1950), condecorado con la Orden del Mérito y la Compañía de Honor, distinguido como Consejero del Rey, y miembro de la Sociedad Real, fue un prominente estadista de Sudáfrica y de la Mancomunidad Británica de Naciones, líder militar y filósofo. Autor de Holism and Evolution: The original source of the holistic approach to life. Londres: Sanford Holst, 1999.

6 Kotler. Philip. El marketing se mueve. Barcelona: Paidós, 2002. p. 14.

7 Íbid. 
El marketing ha cambiado el paradigma y pasó de estar centrado en el producto para centrarse en el cliente. Llamamos a esto concepto de marketing. Ahora, el marketing está tomando un concepto más holístico que tiene que ver con la planeación de los recursos de una empresa, enterprise resource planning (ERP), gestión de cadena de abastecimiento (SCM) y la gestión de relaciones con el cliente (CRM). El cambio emergente consiste en entender el marketing como el proceso de encontrar, crear y agregar valor para el cliente. Y esto solamente puede suceder si todas las funciones están alineadas con la posibilidad de crear valor para el cliente $^{8}$.

\section{¿Qué es un ERP?}

El ERP es un sistema integral de gestión empresarial que está diseñado para modelar y automatizar la mayoría de procesos en la empresa (área de finanzas, comercial, logística, producción, etc.). Su misión es facilitar la planificación de todos los recursos de la empresa.

Kumar y Hillengersberg ${ }^{9}$ definen al ERP como "paquetes de sistemas configurables de información dentro de los cuales se integra la información a través de áreas funcionales de la organización". Los sistemas ERP son extremadamente costosos, y una vez que los sistemas ERP se implantan con éxito, traen una serie de beneficios importantes para las empresas.

Orton y Marlene (2004) ${ }^{10}$ definen los sistemas de planeación de recursos empresariales ERP como sistemas que permiten coleccionar y consolidar información a través de la empresa.

Reuther, D. (2004) en su artículo "Critical Factors for Enterprise Resources Planning System Selection and Implementation Projects within Small to Medium Enterprise" 11 menciona que K.C. Laundon y J.P. Laundon, (2000) definen los sistemas de planeación de recursos empresariales ERP como sistemas de administración de negocios que integran todas las facetas del negocio, incluyendo la planeación, la manufactura, las ventas y las finanzas. El ERP planea y automatiza muchos procesos con la meta de integrar información a lo largo de la empresa y elimina los complejos enlaces entre los sistemas de las diferentes áreas del negocio.

Lo más destacable de un ERP es que unifica y ordena toda la información de la empresa en un solo lugar, de este modo cualquier suceso queda a la vista inmediatamente, posibilitando la toma de decisiones de forma más rápida y segura, acortando los ciclos productivos. Con un ERP tendremos la empresa bajo control e incrementaremos la calidad de nuestros servicios y productos. La implantación de un ERP conlleva la eliminación de barreras interdepartamentales, la información fluye por toda la empresa eliminando la improvisación por falta de información. La planeación de los recursos de la empresa es una evolución de los sistemas MRP, los cuales estaban enfocados únicamente a la planificación de materiales y capacidades productivas. Los ERP disponen de herramientas para efectuar la planificación de los trabajos en planta.

Los ERP más completos ofrecen módulos para planificar a capacidad finita. Los ERP son el núcleo de otras aplicaciones como pueden ser la gestión de las relaciones con los clientes CRM, (Conversión de datos en información útil Data Mining, etcétera.)

Los sistemas unifican información de las diferentes áreas (finanzas, recursos humanos, ventas, manufacturación, etc.) de la empresa en un solo lugar, haciendo más fácil la toma de decisiones dentro de la empresa. El ERP planea y automatiza muchos procesos para integrar información a lo largo de la empresa y eliminar los complejos enlaces entre los sistemas de las diferentes áreas del negocio.

En la actualidad las tecnologías de información juegan un papel importante en las estrategias de negocios, ya que están cambiando la forma como las empresas realizan sus procesos. Los sistemas de información permiten a las compañías lograr ventajas competitivas de diferentes maneras: coordinando actividades de valor en localidades que se encuentran en una amplia geografía, o también mediante la creación de nuevas interrelaciones entre los negocios, ampliando el alcance de las industrias.

8 Entrevista a Philip Kotler. "Cómo crear valor para el cliente”. En: http://www.clarin.com/suplementos/economico/2001/06/10/n-277928. htm

9 Kumar, K., y Hillegersberg, J. v. Enterprise resource planning: Introduction. 2000. Disponible en: www. delivery.acm.org.millenium. $10 \mathrm{http} / / /$ www.findarticles.com/p/articles/mi_qa3993/is_200409/ai_n9449287.

11 Reuther, D., y Chattopadhyay, G. (2004). Critical factors for enterprise resources planning. System selection and implementation projects within small to medium enterprises. 2 851-855 Vol.2 IEEEXplorer [on-line database]. 
Asimismo le sirve a las empresas para soportar sus estrategias competitivas, ya sea para ir un paso delante de la competencia o reducir las ventajas que la misma pueda presentar.

\section{¿Qué es una SCM?}

La gestión de la cadena de suministros (SCM por sus siglas en inglés: Supply Chain Management), está surgiendo como la combinación de la tecnología y las mejores prácticas de negocios en todo el mundo. Las compañías que han mejorado sus operaciones internas ahora están trabajando para lograr mayores ahorros y beneficios al mejorar los procesos y los intercambios de información que ocurren entre los asociados de negocios.

Una exitosa cadena de suministros entrega al cliente final el producto apropiado, en el lugar correcto y en el tiempo exacto, al precio requerido y con el menor costo posible.

La cadena de suministros (SCM) agrupa los procesos de negocios de múltiples compañías, así como a las diferentes divisiones y departamentos de nuestra empresa. Definida de una forma sencilla, SCM engloba aquellas actividades asociadas con el movimiento de bienes desde el suministro de materias primas hasta el consumidor final. Esto incluye la selección, compra, programación de producción, procesamiento de órdenes, control de inventarios, transporte, almacenamiento y servicio al cliente. Pero, lo más importante es que también incluye los sistemas de información requeridos para monitorear todas estas actividades.

La gestión de la cadena de abastecimiento es la coordinación, programación, adquisición, producción, inventarios y entrega de los productos y servicios a los clientes.

\section{¿Qué es CRM?}

CRM (Customer Realtionship Management) es básicamente la respuesta de la tecnología a la creciente necesidad de las empresas de fortalecer las relaciones con sus clientes.
Las herramientas de gestión de relaciones con los clientes CRM son las soluciones tecnológicas para conseguir desarrollar la teoría del marketing relacional. El cual se puede definir como "la estrategia de negocio centrada en anticipar, conocer y satisfacer las necesidades y los deseos presentes y previsibles de los clientes"12.

El presente texto pretende relacionar el concepto de marketing holístico, con el desarrollo de la actividad de prestación de servicios de salud en Colombia, mostrando la situación de cobertura, el nivel de servicio antes de la Ley 100 de 1993, (ver Cuadro 1); la posibilidad de los inversionistas privados en la participación del mercado de prestación de servicios de salud y la aparición de las EPS, y como ellas, implementando el marketing holístico participan o no del aumento de cobertura y del mejoramiento del nivel de prestación de servicios de salud en Colombia. (ver Cuadro 2).

Se inicia, entonces, la descripción de la situación anterior al año 1993, cuando debido a la baja cobertura y calidad de los servicios de salud del ISS, fueron surgiendo otras modalidades de aseguramiento. De manera paralela se desarrolló un subsistema de cajas de previsión para los empleados públicos y de cajas de compensación familiar (CCF). Los empleadores cotizaban el 4\% sobre la nómina, independientemente del número de hijos que tuvieran sus empleados, y las CCF se encargaban de redistribuir los subsidios familiares en proporción al tamaño de la familia de cada trabajador.

Entre 1975 y 1982 creció y se mejoró la planta de hospitales y centros de salud de este subsistema. No obstante, a partir de la crisis fiscal acaecida en ese último año, los recursos que se asignaron a este tipo de servicios se redujeron de $8 \%$ del presupuesto nacional a menos de $4 \%$ en los años sucesivos, lo que ocasionó el progresivo deterioro del sector. A este subsistema le correspondía atender en 1993 aproximadamente el $70 \%$ de la población nacional; sin embargo, su cobertura era muy limitada y la calidad de sus servicios muy deficiente.

Alrededor del 25\% de la población nacional no tenía acceso a ningún tipo de servicios, por lo que gran parte de sus necesidades de salud y de medicamentos estaban siendo paulatinamente cubiertos por los médicos, laboratorios y farmacias del sector privado.

12 Abad, Raúl. Marketing relacional, futuro relacional. Barcelona: Espasa - Carpe, 2003. 
El Sistema Nacional de Salud SNS funcionaba de manera desarticulada y enfrentaba enormes dificultades para hacer realidad el derecho de todos los colombianos a la protección de la salud consagrado en la Nueva Constitución de 1991.

Los objetivos del Sistema General de Seguridad Social en Salud son: regular el servicio público esencial de salud y crear condiciones de acceso de toda la población al servicio en todos los niveles de atención.

Mediante la Ley 100 de 1993, el gobierno creó un conjunto de condiciones que pretendían facilitar una total accesibilidad de la población al servicio de salud.
El cálculo de las utilidades de 16 EPS que se encontraban en diciembre de 1996 agremiadas en ACEMI (Asociación Colombiana de Empresas de Medicina Prepagada y EPS) muestra que mientras en 1995 las pérdidas fueron de $\$ 7.373$ millones, el año anterior se elevaron a \$33.138 millones. Se ha ido abriendo una brecha entre el ritmo al cual aumentan los ingresos -determinados por el nivel de las UPC que fija el gobierno- y aquél al cual se incrementan los costos de las drogas y los servicios de salud, recogidos por el índice de precios al consumidor del DANE. Otro estimativo, el de ingresos y gastos por usuario año señala que, en promedio, cada afiliado implicó una pérdida, para las EPS, de $\$ 24.560$ en el año pasado ${ }^{13}$.

Cuadro 1. Situación del Sistema Nacional de Salud antes de 1993

\begin{tabular}{|c|c|c|c|c|c|}
\hline \multicolumn{7}{|c|}{ Cobertura de afiliación SGSSS } \\
\hline Sistema de Salud Pública & Sistema de Seguros Sociales & Subsistema Privado de Salud \\
\hline$\%$ & Nivel de Servicio & $\%$ & Nivel de Servicio & $\%$ & Nivel de Servicio \\
\hline $40 \%$ & Bajo & $20 \%$ & Medio & $5 \%$ & Alto \\
\hline
\end{tabular}

Fuente: Mabel Lucila Munar Cardozo. "Acreditación de las EPS y sistema de calidad de salud en Colombia y la relación costo - beneficio desde el punto de vista de los usuarios”. Consultado en http://www.monografias.com/

El numeral 4 del artículo 153 de la Ley se refiere al concepto de libre escogencia. El Sistema General de Seguridad Social en Salud permitía entonces la participación de diferentes entidades que ofrecieran la administración y la prestación de los servicios de salud, bajo las regulaciones y la vigilancia del Estado, y aseguraba a los usuarios libertad en la escogencia de las entidades promotoras de salud y de las instituciones prestadores de servicios de salud, cuando ello fuera posible según las condiciones de la oferta de servicios.

Tan sólo tres años después de la implementación de la Ley y la creación de las EPS sus finanzas eran preocupantes:
En 1999, las EPS privadas lograron el punto de equilibrio. Con estrategias de negocio distintas, cada empresa se consolida y amplía su participación en el número de afiliados. ${ }^{14}$

Las entidades promotoras de salud (EPS) privadas tenían ahora sí cómo demostrar que el negocio de la seguridad social les estaba funcionando. 1999 les dejó utilidades cercanas a los $\$ 36.000$ millones y 1’850.000 nuevos afiliados.

Entre 1993 y el año 2000, la cobertura de la seguridad social en salud aumentó del 13\% al 58\% de la población en Colombia. Esto se logró bajo el sistema generado por la Ley 100, dentro del cual han operado

13 Revista Dinero. Sección Negocios.”¿Son viables las EPS?” . Edición 49. Enero 8 de 1997. p. 78-82.

14 Revista Dinero. Sección negocios. "La salud se consolida". Edición 106. Abril 28 de 2000. p. 79-82. 
armoniosamente el sector público y el privado, el sistema contributivo y el subsidiado, en una conjunción de esfuerzos poco común en el concierto mundial. Esta ha sido una tarea de todos. Entre 1994 y 1999, las familias cotizaron US $\$ 5.000$ millones adicionales en el régimen contributivo, al tiempo que el presupuesto público aumentó en US\$4.600 millones para subsidiar la cobertura de los sectores menos favorecidos ${ }^{15}$.

La aparición de las EPS y esta condición de mercado de libre escogencia es la que abre el espacio para el desarrollo de actividades de marketing, surgen, entonces, objetivos de adquisición de pacientes (clientes), mantenimiento de los mismos (fidelización), objetivos de participación de mercado que crean una competencia acorde con el interés del gobierno de lograr una mayor cobertura y mejor prestación de servicios de salud. Objetivos que esperan lograrse apoyados por estrategias organizacionales basadas fundamentalmente en tres pilares: una clara cultura orientada al servicio; una nueva relación contractual de las entidades prestadoras de servicios de salud y del recurso humano competente; y la tecnología de información como elemento integrador de todas las actividades tanto científicas como administrativas de las entidades.

El marketing holístico relaciona las anteriores variables en la medida en que las involucra en los sistemas de planificación de los recursos de una empresa (ERP), gestión de cadena de abastecimiento (SCM), gestión de relaciones con el cliente (CRM). Las EPS las han acogido e implementado en el desarrollo de sus actividades.

\section{El marketing holístico y las entidades prestadoras de servicios de salud}

Se tomaron ejemplos de algunas EPS y otras entidades que hacen parte de la oferta del sector de la salud, para mostrar cómo se ha aplicado el marketing holístico y sus herramientas tecnológicas en la generación de valor de uso y valor de cambio ( beneficios para el usuario del servicio y beneficios para los dueños y empleados de la empresa respectivamente).

Se muestra una serie de necesidades que tenían las EPS, identificándolas y posteriormente las soluciones dadas mediante la integración de paquetes informáti- cos que integran las variables del marketing holístico y los beneficios conseguidos.

\section{Necesidades}

Microsoft Colombia presenta en su página de internet (http://www.microsoft.com) testimonios de empresas que han implantado exitosamente soluciones para responder a necesidades específicas de negocio. ("Colombia, casos de éxito"). Es el caso de la organización Sanitas y de Coomeva que se describen a continuación:

- La organización Sanitas Internacional requería de una aplicación que potenciara su sistema médico-administrativo y su red de servicios de salud. En el mercado no existía un metodo que se ajustara a las necesidades y a la visión de la entidad, por lo que se optó por desarrollar internamente una herramienta que de manera ágil, robusta y administrable, le permitiera a la organización consolidar, analizar y aprovechar la información que a diario generaba, así como prestar a sus profesionales afiliados una solución útil para su labor con los pacientes de la entidad.

- Coomeva requería de la implementación de servicios de atención al cliente y al afiliado, que les permitiera la captura de información y prestación de soluciones en todas las oficinas, IPS y centros de EPS de la cooperativa, que agilizaran procesos y productividad. Unido a una solución que les permitiera pasar al modelo cliente servidor, de manera rápida, eficaz, con bajo costo y sin que les significara gastos ocultos, ni grandes inversiones en renovación tecnológica.

- Clínica Fundación Santa Fé de Bogotá16,17 se propuso disminuir errores en la práctica médica y los sobrecostos en salud. Así como el incremento en la creación de las páginas web transaccionales de los laboratorios farmacéuticos que ponen la información y la educación en salud al alcance de médicos y pacientes.

- Laboratorios Merck Sharp \& Dohme ${ }^{18}$ en Colombia comprometidos con su misión social no solo de traer al país medicamentos innovadores para mejorar la salud y calidad de vida de la población colombiana, consideran importante proporcionar información sobre enfer-

15 Revista Dinero. Sección Agenda Pública. "Colombia modelo mundial de salud”. Edición 119. Octubre 13 de 2000. p. 104.

16 Sánchez, Gina Química Farmacéutica. "Casos ejemplares del uso de la tecnología en el sector salud en Colombia Centro de Medios Independientes de Colombia”. El artículo original está disponible en: http://colombia.indymedia.org/news/2006/04/41413.php

17 Revista Dinero. "Estrategia de negocios. El hospital digital. Fecha: 03/17/2006 - Edición: 249. p. 158.

18 Sánchez, op.cit., p. 15. 
medades, como asma, calvicie, osteoporosis, enfermedades cardiovasculares y SIDA ente otras, dándoles la posibilidad a los pacientes de enviar sus preguntas sobre tratamientos y recibir respuestas de profesionales, emplear calculador as interactivas personalizadas para riesgo cardiovascular, glaucoma y osteoporosis.

Consolidar, analizar y aprovechar la información que a diario se genera, prestar a sus profesionales afiliados una solución útil para su labor con los pacientes de la entidad, implementar servicios de atención al cliente y al afiliado, permitir captura de información y prestación de soluciones en todas las oficinas, IPS y centros de EPS, agilizar los procesos, mejorar los niveles de productividad, disminuir errores en la práctica médica y sobrecostos, proporcionar información pertinente y oportuna a los clientes son formas de entregar valor, posible gracias a la implementación y desarrollo del marketing holístico en las entidades prestadoras de servicios de salud.

\section{¿Cuales fueron las soluciones? ¿Cómo se puso en marcha el "marketing holístico"?}

A partir de la tecnología y la metodología de desarrollo Microsoft, la organización creó para Sanitas a Sophia, un sistema inteligente de 16 módulos, el cual controla efectivamente información de tipo administrativa y médica, lo que le ha permitido a la entidad mejorar su sistema de prestación de servicios de salud, así como aumentar la productividad del negocio. Sophia consolida de manera segura y robusta información de tipo médico como historias clínicas, controles y diagnóstico, así como aspectos de tipo administrativo y asistencial, como citas, cirugías, proveedores y puntos de atención.

Coomeva implementó una estructura con Microsoft Windows Terminal Services, que opera en una granja de diez servidores en donde reside la base de datos de la entidad.

Más de 800 computadores personales en todo el país acceden a los servicios de la plataforma, sin problema, a pesar de que no todos son de última generación. Igualmente corren la aplicación interna de la entidad, así como servicios de la intranet e internet.

En los últimos cuatro años, la Fundación Santa Fe ha invertido US\$4 millones en recurso humano, infraestructura y software para poner en marcha la aplicación de lo que se conoce como e-health (e-salud) que se basa en la utilización de las herramientas de información y comunicación para transformar los procesos asistenciales y educativos, reducir costos y disminuir los errores que se generan cuando no se cumplen los protocolos médicos. La fundación ha desarrollado una red inalámbrica de 400 computadores portátiles con un software que permite optimizar la información y llevar las historias clínicas electrónicas de los pacientes en tiempo real, utilizando la tecnología móvil disponible como teléfonos celulares, PDA e incluso iPod para acceder a la información y para los programas de educación continuada.

Se espera que el manejo de las historias clínicas electrónicas disminuya los costos asistenciales que se pueden presentar por fallo terapéutico por el uso concomitante de medicamentos que interactúan entre sí, en eventos adversos por el uso de medicamentos en patologías contraindicadas, y por el uso irracional de antibióticos, entre otros. También facilitará el seguimiento del paciente y el control de pacientes poli medicados y crónicos.

Se estima que la mayoría de los errores médicos no redunda en lesiones graves o muerte, sino que tienen consecuencias que pasan inadvertidas dado el estado clínico del paciente, pero son sumamente costosos para los sistemas de salud.

Los procesos de sistematización disminuyen el error médico en la práctica habitual. El desafío es lograr que las instituciones de salud de nuestro territorio puedan ser más eficientes cumpliendo con los más altos estándares de calidad ayudándolas en su desarrollo y posterior rentabilidad en la utilización de soluciones de clase mundial.

Las entidades han invertido en mejorar su infraestructura en tecnologías de la información y comunicaciones y de esta manera, lograr un mejor control de sus procesos consiguiendo mayor rentabilidad, eficiencia y eficacia en su administración y garantizando un mejor servicio para los clientes.

Vemos cómo de esta manera convergen salud, tecnología y comercio. La medicina moderna de la más alta calidad se caracteriza por tres elementos fundamentales: un cuerpo médico de primera categoría cuidadosamente seleccionado; tecnología de punta; y estándares que garanticen la seguridad del paciente, para los que, la digitalización juega un papel central. Como vimos, Colombia ya entró en esta tecnología, que con seguri- 
dad favorecerá el desarrollo económico del país gracias a la capacidad para asimilar la transferencia tecnológica del exterior y para adaptarse a nuevas tecnologías de comunicaciones e informáticas. Aunque aún nos encontramos en etapas tempranas de desarrollo tecnológico, sí debe enorgullecernos que el sector de la salud en Colombia no se escapa a la aplicación de los avances tecnológicos como estrategia de competitividad y en busca de mejorar la calidad de la salud que es un derecho constitucional de la población. Es un hecho que la oferta de servicios de salud y educación en salud por medios electrónicos cambiará la práctica médica en el mundo.

\section{Conclusiones}

Es evidente que la mercadotecnia, a través del tiempo, ha ido incursionando cada vez más en el área de servicios, bajo diferentes denominaciones, pero con un objetivo claro, el cual es la identificación, creación, comunicación y entrega de valor tanto de uso como de cambio. Conceptos generales como marketing de servicios, y particulares como marketing sanitario nos acercan a una realidad cada vez más evidente de implementación de estrategias de marketing en las entidades prestadoras de servicios de salud. El marketing holístico desde la perspectiva de Philip Kotler integra la planeación de los recursos de una empresa (ERP), la gestión de la cadena de abastecimiento (SCM) y la gestión de relaciones con el cliente (CRM), como soluciones informáticas independientes o integradas en un solo paquete sistematizado y hacen, cada vez más, parte del desarrollo y administración de las actividades necesarias para una eficiente prestación de servicios de salud.
Los siguientes son los indicadores de cobertura del año 2000 al 2006 que presentó el Foro Nacional "Evolución de Indicadores de Salud", en septiembre 28 de 2006, en la Fundación Santa Fe de Bogotá. Esta información se encuentra en: http://www.asivamosensalud. org/descargas/Carrasquilla_Indicadores.pdf.

En la Revista Dinero, sección Agenda Pública, en el articulo "Colombia modelo mundial de salud", edición 119 de octubre 13 de 2000, en la página 104, se menciona como entre 1993 y 2000, la cobertura de la seguridad social en salud aumentó del 13\% al 58\% (56\% en el Cuadro No. 2), de la población en Colombia. Esto se logró con el sistema generado por la Ley 100, dentro del cual han operado armoniosamente el sector público y el privado, el sistema contributivo y el subsidiado, en una conjunción de esfuerzos poco común en el concierto mundial.

En las variaciones se ve claramente que el régimen contributivo llega en el año 2000 a un 33,5\% de cobertura y de ahí en adelante hasta el 2006 mantiene una tendencia alrededor del 30\%; La implementación de herramientas tecnológicas promovida por las entidades privadas (EPS), no ha incidido en una mayor cobertura en los últimos cinco años.

La variación en el régimen subsidiado llega casi a doblar la cobertura, pasando de $22.47 \%$ al $40.36 \%$.

La aplicación del marketing holístico, ha generado valor a las entidades prestadoras de servicio, optimizando procesos internos, reduciendo errores y en consecuencia reduciendo costos.

Nos queda preguntarnos como usuarios, ¿qué tanto ha mejorado el nivel de calidad en la prestación del servicio?

\section{Cuadro 2. Evolución de Indicadores de Salud}

\begin{tabular}{|l|c|c|c|c|c|c|}
\hline Indicadores & 2000 & 2001 & 2002 & 2003 & 2004 & 2005 \\
\hline $\begin{array}{l}\text { \% de cobertura por afiliación } \\
\text { al régimen contributivo }\end{array}$ & 33.5 & 31.0 & 30.0 & 31.0 & 32.8 & 33.7 \\
\hline $\begin{array}{l}\% \text { de cobertura por afiliación } \\
\text { al régimen subsidiado }\end{array}$ & 22.47 & 25.7 & 26.11 & 26.62 & 34.4 & 40.36 \\
\hline$\%$ total afiliados & $\mathbf{5 6 . 0}$ & $\mathbf{5 6 . 7}$ & $\mathbf{5 6 . 1}$ & $\mathbf{5 7 . 6}$ & $\mathbf{6 7 . 1}$ & $\mathbf{7 4 . 1}$ \\
\hline
\end{tabular}

* Porcentaje de subsidios parciales sobre el total de afiliaciones a régimen subsidiado.

Fuente: Foro Nacional "Evolución indicadores de salud”. Fundación Santa Fe. Septiembre 28 de 2006. 


\section{Bibliografia}

- Grönroos Christian. Strategic Management and Marketing in the Service Sector. Cambridge, Mass Marketing Science Institute, 1983.

- Grönroos Christian. Marketing y gestión de servicios. Madrid, Díaz de Santos, 1994.

- Iturralde, Ernesto. El pensamiento lateral, vertical y holístico. http://www.yturralde.com/plateral.htm. 2007.

- Kotler, Philip. El marketing se mueve. Barcelona, Paidós. 2002.

- Lamata F., Conde J., Martínez B., Horno M. Marketing Sanitario. Madrid, Díaz de Santos, 1994.

- March Cerdá J.C. "La salud es transmisible: Marketing Social y de Servicios." En: Aranda Regules J.M. (Editor). Nuevas perspectivas de atención primaria de salud: Una revisión de la aplicación de los principios de AlmaAta. Madrid, Díaz de Santos, 1994.

- Reuther, D., y Chattopadhyay, G. (2004). Critical factors for enterprise resources planning. system selection and implementation projects within small to medium enterprises. 2 851-855 Vol.2 IEEEXplorer [on-line database].

- Revista Dinero. Sección Negocios. “Son viables las EPS?” Edición 49. Enero 8 de 1997.

- Revista Dinero. Sección Negocios. "La salud se consolida.” Edición 106. Abril 28 de 2000.

- Revista Dinero. Sección Agenda Pública. "Colombia modelo mundial de salud." Edición 119. Octubre 13 de 2000.

- Revista Dinero. Estrategia de negocios. "El hospital digital”. Edición: 249. Marzo 16 de 2006.

- Sánchez, Gina."Casos ejemplares del uso de la tecnología en el sector salud en Colombia Centro de Medios Independientes de Colombia”. El artículo original está http://colombia.indymedia.org/ news/2006/04/41413.php 